\title{
Cuadro clínico de inicio de la diabetes tipo 1 en el niño
}

\author{
María Isabel Hodgson B, Juan Cristóbal O ssa A, Nicolás \\ Velasco F, Pascuala U rrejola N, Antonio Arteaga LI.
}

\section{Clinical picture at the onset of type 1 diabetes mellitus in children}

Background: Type 1 diabetes mellitus (DM1) is a disease of increasing incidence among children. The time elapsed between the beginning of symptoms and the diagnosis of the disease is, in most cases, very extended. Aim: To report the clinical picture at onset and laboratory features of children with DM1. Material and methods: Retrospective review of all medical records of patients admitted to the hospital with a DM1 of recent onset. Results: Sixty three males aged $95 \pm 47$ months and 34 females aged $109 \pm 51$ months, were studied. Males were significantly younger than females $(p<0.05)$. The lapse between symptoms onset and the diagnosis of the disease was longer in girls than in boys $(46 \pm 46$ and $26 \pm 26$ days respectively, $p<0.02)$. There was an inverse correlation between plasma glucose and the lapse between symptoms onset and the diagnosis of disease. The most common clinical picture of the disease was the classical symptoms of diabetes (polyuria, polydipsia, weight loss), in $60 \%$ of cases. Thirty seven percent had developed ketoacidosis at the time of the diagnosis. Ketoacidosis was more common between 1988 and 1995, as compared with the period 1996-2003 (50\% and 28.8\%, respectively). The onset of symptoms occurred between April and August (Winter in the Southern Hemisphere) in 38\% of cases. Conclusions: DM1 is a disease of increasing incidence among children, whose diagnosis is delayed, even though symptoms are evident. The lower incidence of ketoacidosis in our patients between 1996 and 2003 is encouraging (Rev Méd Chile 2006; 134: 1535-40).

(Key w ords: Diabetes complications; Diabetes mellitus, type 1; Ketoacidosis, diabetic)

Recibido el 3 de enero, 2006. Aceptado el 10 de mayo, 2006.

Departamentos de Pediatría y de Nutrición, Diabetes y Metabolismo. Facultad de Medicina, Pontificia Universidad Católica de Chile. Santiago de Chile.

L a diabetes mellitus tipo 1 (DM1), es una enfermedad crónica de manejo complejo, que causa serias alteraciones metabólicas las que, si no son eficazmente controladas, provocarán variadas complicaciones anatómicas y funcionales, luego

Correspondencia a: Dra. María Isabel Hodgson B. Departamento de Nutrición. Alameda 340, Santiago de Chile. Fax: (562) 6338298. E mail: hodgson@med.puc.cl de algunos años de exposición a la enfermedad. Cuando comienza en la infancia, la DM1 agrega a todo lo dicho previamente, un serio conflicto familiar ${ }^{1}$.

La incidencia de la DM1 en niños parece estar aumentando, tanto en Chile como en otros paí$\mathrm{ses}^{2-8}$. De hecho, en nuestro país, dicha incidencia se ha duplicado en 15 años. Según lo publicado por Carrasco et al, en 1990 esta incidencia era de 
poco más de 2,0/100.000 menores de 15 años. En el año 2006, los mismos autores comunican una incidencia de 4,02/100.000. Esta última cifra, a pesar de ser el doble de la de 1990, es bastante inferior a las reportadas en países como Finlandia, que tiene incidencias superiores a los 40,0/ $100.000^{2,3}$.

A pesar de su moderada incidencia, la diabetes es una enfermedad que demanda un manejo constante y crónico. Dado el aumento de la incidencia, la prevalencia de esta enfermedad irá aumentando hasta una cifra que hará inevitable que muchos pediatras tengan algún contacto con pacientes portadores de DM1.

El cuadro clínico de la DM1 en niños es muy similar al de los adultos y el diagnóstico oportuno de la enfermedad permite iniciar el manejo metabólico, antes que sobrevenga la cetoacidosis, complicación aguda que exige un gran esfuerzo terapéutico y que puede ser mortal ${ }^{1}$.

Por todo lo anterior, es importante conocer las características clínicas y los diferentes modos como se presenta esta enfermedad, para estar alerta y no retrasar su diagnóstico.

El objetivo de este trabajo es analizar el modo de comienzo y las características clínicas y de laboratorio de una serie consecutiva de 97 niños que inician una DM1.

\section{MATERIAL Y MÉTODOS}

Desde enero de 1988 hasta enero de 2003, ingresaron al Hospital Clínico de la Pontificia Universidad Católica de Chile 97 pacientes portadores de DM1 que estaban iniciando su enfermedad. Para los fines de la presente comunicación, se efectuó una revisión retrospectiva de las fichas clínicas de todos los pacientes y se registraron los datos demográficos generales (edad, sexo), la edad de inicio de la enfermedad, el tiempo que medió entre el inicio de los síntomas y el diagnóstico, los síntomas de mayor relevancia en cada caso, la glicemia de inicio (técnica semiautomática de hexoquinasa, Hitachi ${ }^{\circledR}$ ), el pH sanguíneo y el bicarbonato del plasma (analizador automático Ciba-Corning $\left.{ }^{\circledR}\right)$, la hemoglobina glicosilada (en ese período se midió Hb A1 según Gabbay $^{9}$, con un máximo normal de 7,5\%), el sodio y potasio plasmáticos (por ión selectivo,
Hitachi ${ }^{\circledR}$ ), la cetonemia plasmática (semicuantitativa), el estado nutritivo evaluado por z score del índice de masa corporal ${ }^{10} \mathrm{y}$ los antecedentes familiares de diabetes.

Se consideró que el paciente presentaba cetoacidosis cuando el pH era igual o menor de 7,3, el bicarbonato menor de 15,0 mmol/l y había cetonas en plasma ${ }^{11,12}$.

Los datos se presentan como promedio (X) \pm desviación estándar (DS). Para el análisis estadístico, se usó el test de $t$ de Student para series no pareadas, el $\mathrm{x}^{2}$ en análisis de frecuencias y la correlación según Pearson entre series de datos. Se consideró significativo un $\mathrm{p}<0,05$.

\section{RESUlTADOs}

Desde enero de 1988 a enero de 2003, ingresaron a nuestro hospital 97 nuevos casos de DM1, los que estuvieron hospitalizados por $8,7 \pm 2,9$ días, siendo luego derivados a control ambulatorio al policlínico de nutrición y diabetes pediátrica de nuestra institución. El $50 \%$ de estos pacientes habían consultado médico previo al ingreso. En sólo la mitad de dichos casos se efectuó el diagnóstico de DM1.

De estos 97 niños, 63 eran varones y 34 mujeres y tenían una edad de $97 \pm 49$ meses (rango: 16 a 192). Los niños eran menores que las niñas, tuvieron un menor período de síntomas previo al diagnóstico (PPDIAG), una glicemia mayor, una hemoglobina glicosilada menor y similar frecuencia de cetoacidosis que las niñas (Tabla 1)

El índice de masa corporal (IMC) fue de $16,8 \pm 2,62$, lo que equivale a un z-score de $0,01 \pm 0,92$ según edad y sexo (rango: $-2,07$ a $+3,07)$. No se observaron diferencias entre hombres y mujeres (hombres z-score $0,08 \pm 1,08$ y mujeres $-0,13 \pm 0,62, p<0,15$, no significativo). Al estratificar según $\mathrm{z}$ score de IMC, 79,2\% eran eutróficos ( $\mathrm{z}$ score de $-1 \mathrm{a}+1$ ), 10,0\% presentaban bajo peso ( $\mathrm{z}$ score menor a -1) y $10,8 \%$ se encontraban con sobrepeso u obesidad (z score mayor a +1). El bajo peso se distribuyó en forma similar en hombres y mujeres. El sobrepeso y la obesidad fueron más frecuentes en varones (hombres $17,3 \%$ y mujeres 4,2\%, p <0,05). El z score IMC correlacionó con la glicemia en forma inversa 


\section{Tabla 1. C aracterísticas demográficas y exámenes de laboratorio en 97 pacientes portadores de D M 1 recién diagnosticada}

\begin{tabular}{|lccc|}
\hline & Total & Hombres $(\mathrm{n}=64)$ & Mujeres $(\mathrm{n}=33)$ \\
\hline Edad (meses) & $97,1 \pm 49,1$ & $94,9 \pm 46,5^{\#}$ & $108,5 \pm 51,2^{\#}$ \\
Período síntomas previo & & & \\
al diagnóstico (días) & $33,1 \pm 35,7$ & $26,2 \pm 26,4^{*}$ & $46,2 \pm 46,3^{*}$ \\
Glicemia (mg/dl) & $497,2 \pm 243,0$ & $529,2 \pm 234,4^{\#}$ & $434,2 \pm 251,7^{\#}$ \\
Hb glicosilada (\%) & $14,4 \pm 2,5$ & $14,0 \pm 2,2^{\#}$ & $15,3 \pm 2,2^{\#}$ \\
Bicarbonato plasma & & & \\
(mmol/l) & $15,4 \pm 6,8$ & $15,5 \pm 6,6$ & $15,4 \pm 6,9$ \\
pH arterial & $7,28 \pm 0,15$ & $7,28 \pm 0,13$ & $7,28 \pm 0,18$ \\
Potasio plasma (mEq/l) & $4,33 \pm 0,66$ & $4,36 \pm 0,66$ & $4,29 \pm 0,71$ \\
Cetoacidosis (\% casos) & 37,1 & 38,1 & 35,3 \\
\hline
\end{tabular}

${ }_{p}<<0,05 .{ }_{p}^{*}<0,02$. Resto de las diferencias no significativas.

$(r=-0,21, p<0,05)$. No se observó correlación del z score IMC con hemoglobina glicosilada u otro parámetro clínico o bioquímico, salvo la glicemia.

En cuanto al potasio plasmático, no se observó ningún caso con potasemia mayor de $6,0 \mathrm{mEq} / \mathrm{l}$ y sólo siete niños presentaron niveles menores de $3,5 \mathrm{mEq} / \mathrm{l}(7,2 \%)$ al ingreso al hospital.

El PPDIAG fue menor en los niños más pequeños. De hecho, los menores de 5 años de edad tuvieron un período previo al diagnóstico de $20,5 \pm 14,5$ días y los mayores de esa edad $39,1 \pm 36,2$ días ( $\mathrm{p}<0,05$ versus menores de 5 años). Al estratificar la duración del PPDIAG, 19,6\% fue menor de 2 semanas, $44,3 \%$ de 2 a 6 semanas y $36,1 \%$ mayor de 6 semanas. Existió una correlación inversa entre glicemia y PPDIAG $(r=-0,33, p$ $<0,004)$ y una correlación directa de la hemoglobina glicosilada con el mismo período $(\mathrm{r}=0,31, \mathrm{p}$ $\varangle 0,001)$. El PPDIAG no correlacionó con $\mathrm{pH} 0$ potasemia, y tampoco con el estado nutritivo.

En cuanto al cuadro clínico, los síntomas más frecuentes fueron polidipsia, poliuria y baja de peso (Tabla 2). Sólo dos pacientes presentaron compromiso de conciencia, calificado como sopor superficial y once tuvieron signos clínicos de deshidratación al momento del ingreso.

En términos generales, se clasificó la forma de presentación de la enfermedad en: cuadro clásico (polidipsia, poliuria, baja de peso) $60,4 \%$ y cetoacidosis $37,5 \%$. Dos casos (2,1\%), fueron considerados como hallazgos de laboratorio.
Como enfermedades asociadas, se pesquisaron cuatro infecciones respiratorias altas y dos bajas (las 2 correspondieron a neumonía por Mycoplasma). Un paciente era portador de síndrome de Down y uno estaba en tratamiento por hipotiroidismo desde seis años previo a la aparición de DM1.

Se constataron antecedentes familiares (primer y segundo grado) de DM1 en 7 casos $(7,2 \%)$ y de diabetes tipo 2 en 44 pacientes (45,4\%).

Los casos diagnosticados entre los períodos 1988-1995 ( $\mathrm{n}=38$ ) y 1996-2003 ( $\mathrm{n}=59$ ), presentaron diferencias en la edad al momento del diagnóstico,

\section{Tabla 2. Síntomas al inicio de la enfermedad en 97} pacientes portadores de D M 1

\begin{tabular}{|lcc|}
\hline Síntoma & $\mathrm{n}$ casos & $\%$ casos \\
\hline Polidipsia & 85 & 87 \\
Poliuria & 83 & 85 \\
Baja de peso & 70 & 71 \\
Polifagia & 36 & 37 \\
Astenia & 25 & 26 \\
Anorexia, náuseas, vómitos & 28 & 29 \\
Dolor abdominal & 14 & 15 \\
Enuresis & 11 & 11 \\
Otros* & 4 & 4 \\
\hline
\end{tabular}

*Un caso de sopor superficial, dos de cefalea, uno con parestesias. 
la distribución por sexo, la duración del PPDIAG, la proporción de menores de 5 años de edad, la incidencia de cetoacidosis y los requerimientos de insulina al alta (Tabla 3).

La mayor proporción de los casos debutó en el período junio-agosto (38,1\%). En los períodos de diciembre-febrero, marzo-mayo y septiembre-noviembre, debutaron $19,6 \%, 24,7 \%$ y $17,6 \%$ de los casos, respectivamente.

\section{DisCUSIÓN}

La experiencia clínica que se presenta en este trabajo permite destacar varios aspectos de importancia. En primer término, el período que medió entre el inicio de los síntomas y el diagnóstico de la DM1 es amplio y, en un porcentaje todavía muy alto, la enfermedad se pesquisó con cetoacidosis. La mayoría de los casos portadores de esta grave complicación podrían haber sido diagnosticados antes de su aparición, pues casi todos presentaban síntomas característicos de la enfermedad. Una proporción importante de estos pacientes (50\%) consultó médico previamente y sólo en la mitad de los casos se efectuó el diagnóstico correcto. De hecho, dos pacientes presentaron síntomas por cuatro meses, sin que se reconociera la DM1 en las dos ocasiones que consultaron médico, ingresando luego en cetoacidosis al hospital. Lo anterior sólo refleja que la DM1 no estuvo plenamente integrada al repertorio diagnóstico de varios de los médicos que atendieron a estos niños, ya que la mayoría de los pacientes presentaban una sintomatología claramente identificable.

Por lo tanto, se debe continuar alertando a los médicos para que incorporen a la DM1 a su repertorio diagnóstico y hagan una anamnesis dirigida cuando la sospechen. De esta manera efectuarán un diagnóstico precoz de la enfermedad. Una meta razonable es acercarse a lo observado en países desarrollados, en donde la incidencia de cetoacidosis al comienzo de la enfermedad es menor al 20\% ${ }^{13}$.

Barker et al ${ }^{11}$, demostraron que el seguimiento de niños en alto riesgo de desarrollar diabetes reduce en más de diez veces la frecuencia de hospitalización y se asocia a una reducción significativa de la glicemia y la hemoglobina glicosilada al momento del diagnóstico, comparados con un grupo de niños con DM1 diagnosticada en un servicio clínico comunitario. Esta experiencia se basa en un complejo seguimiento de niños con predisposición genética y anticuerpos anti-islotes (+), poco repetible en la práctica clínica. A pesar de lo anterior, se puede concluir de ella que esta «alerta clínica»frente a la DM1 hace más precoz el diagnóstico y evita complicaciones agudas. Esta situación es similar a la observada en dos de nuestros pacientes, que iniciaron su enfermedad clínica en el período que estaban bajo control seriado, pues formaban parte de un protocolo de estudio de prevalencia de ICA (+) en parientes de primer grado de diabéticos tipo $1^{14}$.

Tabla 3. D iferencias entre los pacientes diagnosticados en el período 1998-1995 y 1996-2003

\begin{tabular}{|lcc|}
\hline & $1988-1995(\mathrm{n}=38)$ & $1996-2003(\mathrm{n}=59)$ \\
\hline $\begin{array}{l}\text { Edad al diagnóstico (meses) } \\
\text { Sexo masculino (\%) }\end{array}$ & $108,3 \pm 40,2^{\#}$ & $89,2 \pm 52,3^{\#}$ \\
$\begin{array}{l}\text { Período síntomático previo al } \\
\text { diagnóstico (días) }\end{array}$ & $55,3^{\#}$ & $72,9^{\#}$ \\
$\begin{array}{l}\text { Menores de 5 años de edad } \\
\text { (\% de los casos) }\end{array}$ & $47,3 \pm 48,2^{* *}$ & $23,6 \pm 19,2^{* *}$ \\
$\begin{array}{l}\text { Incidencia cetoacidosis (\%) } \\
\text { Dosis de insulina al alta } \\
\text { (U/k peso) }\end{array}$ & $13,2^{* *}$ & $35,6^{* *}$ \\
\end{tabular}

$\#_{p}<0,05$. **p $<0,01$. 
Los datos disponibles en nuestro medio con respecto al cuadro clínico de inicio de DM1 en niños son escasos. Arteaga, en una serie clínica no publicada, resumió observaciones de 65 niños portadores de DM1 reclutados entre 1960 y 1976. De ellos, 43\% iniciaron su enfermedad en cetoacidosis (A. Arteaga, comunicación personal). Mella ${ }^{15}$, en una publicación de 1985, relató que: el coma diabético ha disminuido (10\%) y se presenta especialmente en menores de 5 años»(sin que se aclare a qué se refiere la autora al usar el término «coma diabético» y que «a duración de síntomas prediagnósticos es variable, siendo más breve en niños pequeños» lo que coincide con nuestra experiencia. Luego, agrega que el período sintomático previo al diagnóstico es de $\ll$ semanas en 30\%, 3 a 6 semanas en $50 \%$ y más de 6 semanas en $20 \%$ de los casos». $\mathrm{Si}$ comparamos nuestros datos con estas experiencias constataremos que, a pesar de haber pasado muchos años, la oportunidad en el diagnóstico de DM1 en niños parece no haber mejorado.

Neu et $\mathrm{al}^{12}$, en una evaluación de 10 años en el estado alemán de Baden-Württemberg, pesquisaron 2.121 niños que debutaron con DM1. En ellos, describieron una incidencia de cetoacidosis (glicemia mayor de $250 \mathrm{~m} / \mathrm{dl}, \mathrm{pH}<7,3$, bicarbonato $<15$ mmol y cetonuria) variable entre $20 \%$ y $31 \%$. Por otra parte, Kulaylat y Narchi ${ }^{16}$, en una serie de 49 pacientes de Arabia Saudita, encontraron 77\% de cetoacidosis en los niños que inician la DM1, a pesar que en esos casos, el PPDIAG promedio fue 12,5 días. Sin embargo, los parámetros diagnósticos usados por estos autores pueden haber abultado las cifras, pues consideraron con cetacidosis a niños con $\mathrm{pH}$ menor de 7,35. En todo caso, los pacientes con un $\mathrm{pH}$ de 7,1 o menos, constituyeron $38 \%$ de los casos. Savova et $\mathrm{al}^{17}$, en 1.248 niños búlgaros con DM1 recién diagnosticada, describieron una incidencia de cetoacidosis «severa» $(\mathrm{pH}$ bajo 7,2 ) de $18,2 \%$. Por último, Levy-Marechal y Czernichow $^{18}$, en 520 nuevos casos de DM1 en jóvenes y niños franceses menores de 19 años, comunicaron una incidencia de $\mathrm{CO}_{2}$ plasmático menor a $18 \mathrm{mmol} / \mathrm{l}$ en $48 \%$ de los casos.

En nuestros pacientes, la menor incidencia de cetoacidosis del segundo período, coincidió con una mayor precocidad en el diagnóstico de la enfermedad. La misma observación ha sido descrita por Neu et $a^{12}$. En este último grupo, la dosis de insulina requerida al alta fue menor. Todos estos argumentos sugieren que, mientras más precozmente se haga el diagnóstico de DM1, menor será la incidencia de cetoacidosis diabética y menores los requerimientos de insulina. Es interesante destacar que el PPDIAG de los varones fue más breve, hecho también descrito por otros ${ }^{16}$. La causa de esto no está clara, ya que la cetoacidosis, uno de los factores que acelera la consulta y el diagnóstico de la DM1 ${ }^{12,17}$, tiene incidencia similar en varones y niñas. Por otra parte, los niños tuvieron mayor glicemia que las niñas y existió una correlación inversa entre glicemia y PPDIAG, lo que sugiere que el diagnóstico es de mayor precocidad porque el cuadro metabólico es de mayor agresividad. Por otra parte, las mujeres tuvieron una hemoglobina glicosilada mayor, a pesar de tener una menor glicemia al momento del diagnóstico, lo que sugiere que el mayor tiempo de exposición a la hiperglicemia, concordante con PPDIAG mayor, es el principal factor que influye en dicha medición.

A pesar de que $71 \%$ de nuestros pacientes acusó baja de peso, el estado nutritivo al ingreso al hospital estaba conservado en la mayoría de los pacientes. No contamos con los datos de peso previo a la baja reportada, por lo que no podemos cuantificarla.

En esta serie, la mayor parte de los pacientes fueron varones. Carrasco et al, en Santiago de Chile, describieron incidencia similar de DM1 en hombres y mujeres ${ }^{2}$. En esa publicación se muestra una distribución por sexo similar en niños de 0 a 4 años, mayor en hombres de 5 a 9 años y mayor en mujeres de 10 a 14. En nuestro caso, sólo $34 \%$ de los pacientes se encontraban entre los 5 y los 9 años, por lo que la mayor proporción de varones no se debería a una concentración en ese rango de edad. Sin embargo, lo comunicado por Carrasco et al en $2006^{3}$, muestra que la incidencia (por cada 100.000 niños menores de 15 años), fue muy similar en ambos sexos hasta el año 2000. De allí en adelante hay una clara predominancia de los varones (año 2001: hombres 6,9, mujeres 5,13; 2002: hombres 6,38, mujeres 4,72 y año 2003: hombres 8,47 y mujeres 6,61 ). Los datos provenientes de otros países muestran incidencias similares en ambos sexos ${ }^{4-6}$ o claramente mayores en varones ${ }^{7,8}$. En conclusión, la DM1 en niños es una enfermedad fácilmente diagnosticable, con una sintomatología similar a lo observado en la DM1 de adultos. El retraso del 
diagnóstico no se debe a que la enfermedad sea difícil de diagnosticar o presente síntomas poco destacados. Por el contrario, estos últimos son claros y fáciles de pesquisar. Es importante, por lo tanto, mejorar la habilidad diagnóstica de los

\section{REFERENCIAS}

1. Bode BW edit. Medical Management of type 1 diabetes. American Diabetes Association. Alexandria 2004.

2. Carrasco E, Pérez F, Calviluán C, López G, WolfF F, CASTAÑO A ET AL. Incidencia de diabetes mellitus insulinodependiente en Santiago de Chile (19901993). Rev Méd Chile 1996; 124: 561-6.

3. Carrasco E, Pérez-Bravo F, Dorman J, Mondragón A, SANTOS JL Increasing incidence of type 1 diabetes in population from Santiago de Chile: trends in a period of 18 years (1986-2003). Diabetes Metab Res Rev 2006; 22: 34-7.

4. Eurodiab Ace Study Group. Variation and trends in incidente of childhood diabetes in Europe. Lancet 2000; 355: 873-6.

5. Haynes A, Coger C, Bubara MK, Jones TW, Davis EA. Continued increase in the incidence of childhood type 1 diabetes in a population-based Australian sample (1985-2002). Diabetología 2004; 47: 866-70.

6. Lupman TH, Yuejang C, Murphy KM. The epidemiology of type 1 diabetes in children in Philadelphia 1990-1994. Evidence of an epidemic. Diabetes Care 2002; 25: 1969-75.

7. Casu A, Pascuto C, Bernardem L. Type 1 Diabetes among Sardinian is increasing. Diabetes Care 2004; 27: 1623-9.

8. Joner G, Stene L, Sovik O. Nationwide, prospective registration of type 1 diabetes in children aged $<15$ years in Norway 1989-1998. Diabetes Care 2004; 27: 1618-22.

9. Gabbay KH, Haty K, Breslow JL, Euson RC, BunN HF, GALOP PM. Glycosylated hemoglobins and long-term blood glucose control in diabetes mellitus. J Clin Endocrinol Metab 1977; 44: 859-64.

10. Central for Disease Control-USA. Nacional Center médicos y educar a la población, para favorecer un diagnóstico más precoz de la DM1 en niños.

Pensar en esta condición facilita el diagnosticarla, evitando serias complicaciones para los niños que comienzan una DM1.

for Health Statistics-BMI for age charts-2000. http://www.cdc.gov/nchs/growthcharts

11. Barker JM, Goehrig SH, Barriga K, Hoffman M, SLover R, EISENBarth GS et aL. Clinical characteristics in children diagnosed with type 1 diabetes through intensive screening and follow-up. Diabetes Care 2004; 27: 1399-404.

12. Neu A, Wiшasch A, Ehehalt S, Hub R, Ranke MB. Ketoacidosis at onset of type 1 diabetes mellitus in children-frequency and clinical presentation. Pediatric Diabetes 2003; 4: 77-81.

13. Rosenbauer J, Icks A, Giani G. Clinical characteristics and predictors of severe ketoacidosis at onset of type 1 diabetes mellitus in children in a NorthRhine-Westphalian region, Germany. J Pediatr Endocrinol 2002; 15: 1137-45.

14. Olmos PR, Hodgson Mi, Maiz A, Manrique M, Díaz de VaLDÉS M, FonCEA R ET aL Nicotinamide protected first-phase insulin response (FPIR) and prevented clinical disease in first-degree relatives of tippe 1 diabetics. Diabetes Res Clin Pract 2006; 71: 320-33.

15. Mela I. Diabetes Infantil. En Veit O, Zavala C edit. Actualización en diabetes mellitus. Series clínicas de la Sociedad Médica de Santiago. Editorial Universitaria, Santiago de Chile 1985.

16. Kulaylat NA, Carchi H. Clinical Picture of childhood type 1 diabetes mellitus in the eastern province of Saudi Arabia. Pediatric Diabetes 2001; 2: 43-7.

17. Savova R, Popova G, Koprivarova K, Konstantinova M, Angelova B, Atasanova M et al. Clinical and laboratory characteristics of type 1 (insulin dependent) diabetes mellitus at presentation among Bulgarian children. Diabetes Res Clin Pract 1996; 34 suppl: S159-S163.

18. Levy-Marechal C, Czernichow P. Heterogeneity of type 1 diabetes at onset in children: results from the French incidence study. Diabetes Metab 1993; 19: 296-303. 